\title{
Coupling of Concanavalin A to Cellulose Hollow Fibers for Use in Glucose Affinity Sensor
}

\author{
Keeran R. Srinivasan, Sohrab Mansouri, ${ }^{*}$ and Jerome S. Schultz \\ Department of Chemical Engineering, The University of Michigan, \\ Ann Arbor, Michigan 48109-2136
}

Accepted for publication May 10, 1985

This article describes a method for the chemical immobilization of concanavalin $A$ (Con $A$ ) on the inside wall of a single hollow cellulose fiber for use in giucose affinity sensor. Periodate oxidation of cellulose fiber followed by a spacer for Con A attachment was deemed to be the most optimal procedure for achieving the highest sensitivity of the sensor without compromising its physical integrity. The effects of variables like the duration of periodate oxidation and its concentration and $\mathrm{pH}$ of the spacer coupling step and its duration have been examined. The mechanical strength of the hollow fiber as well as its permeability to the analyte (glucose) have been evaluated prior to and after Con A coupling process.

It has been demonstrated that Con A bound hollow fiber prepared according to the procedure outlined here can be successfully used to construct glucose affinity sensor for operation in the physiological range of glucose concentrations.

\section{INTRODUCTION}

The idea of an affinity sensor was first proposed by Schultz and Sims. ${ }^{1}$ It was based on competitive equilibrium binding between two ligands, one of which is the metabolite or hormone of interest, to a protein immobilized on an inert matrix. The binding site on the protein is equally accessible to both ligands, but their affinities to the site are usually different. If the second ligand is suitably tagged with a fluorescent dye like fluorescein, its displacement from the protein can be measured optically, and the modulation of this displacement can be related to the changing levels of the first ligand.

An affinity sensor based on this principle was built by Schultz et al. ${ }^{2}$ for the measurement of glucose concentration in plasma. Concanavalin A (Con A) immobilized on the inside surface of a hollow dialysis fiber by glutaraldehyde cross-linking was used as the receptor protein and fluorescently labeled dextran (FITC-dextran, average molecular weight, 70,000 daltons) as the competing ligand. Though the sensor

\footnotetext{
* Present address: Cardiac Pacemakers Inc., 4100 No. Hamline Avenue, St. Paul, MN 55164.
}

worked quite satisfactorily, initial experiments indicated that the sensitivity was not reproducible, and the longterm stability left much to be desired. It was recognized that chemical immobilization of Con $A$ to the inside wall of the cellulosic fiber could be a major improvement in the development of a workable sensor.

There is no doubt that an implantable blood glucose sensor with the required levels of sensitivity, longterm stability, and temporal response would be a major step forward in the management of patients. It is difficult to overstate the usefulness of such a real-time monitoring device during the surgery of critically ill patients. The continuous nature of the output of such a device raises the possibility that, in the near future, it could become the feedback control element in a closed-loop system designed to maintain nearly constant levels of blood glucose. In other words, a reliable and implantable blood glucose sensor is an important component of an artificial pancreas. Finally, it is anticipated that an implantable glucose sensor with a much wider dynamic range of detection can be used in the fermentation industry for process control.

Many methods for covalent bonding of proteins and enzymes to cellulose are known, ${ }^{3,4}$ though most of these relate to immobilization on cellulose powder or beads. Only recently have methods emerged for use with a bundle of hollow dialysis fibers..$^{5-9}$ The difference in these methods is in the use of activating reagents. For example, Jackson et al. ${ }^{5}$ used $\mathrm{CNBr}$, and Dumler et al. ${ }^{6}$ preferred the trichloro-s-triazine (TsT) method. Mazzola et al. ${ }^{7,8}$ used a combination of the above methods plus glutaraldehyde cross-linking. Finally, periodate oxidation was chosen by Rossi et al. ${ }^{9}$ to link arginase to cuprophan (a type of cellulose) hollow fibers. In this study we report on the immobilization of Con A on the inside wall of a single hollow fiber. In keeping with our long-term objectives, we required the method to (1) maximize the amount of Con A bound to the fiber, (2) minimize the loss of reactivity of Con A toward its natural ligands, and (3) minimize the damage to the fiber itself. 
Initial experiments were done with finely chopped fibers. The optimal procedure was then applied to single fibers. Finally, the mechanical strength of the fiber, its permeability to small molecules before and after immobilization, and the long-term stability of bound Con $\mathrm{A}$ have been examined.

On the basis of our experience as well as the wellknown problems associated with $\mathrm{CNBr}$ activation (e.g., the slow leaching of bound ligand in presence of nucleophiles ${ }^{10}$ and the fact that the activated sorbent acquires anion exchange properties at neutral $\mathrm{pH}^{11,12}$ ), we chose periodate oxidation of Sanderson and Wilson ${ }^{13}$ as the method to activate cellulose fiber.

\section{MATERIALS AND METHODS}

Initial experiments were done with chopped fibers from a Cordis-Dow (C-DAK) artificial kidney. Single fiber runs were carried out using Enka hollow fibers.

Both fibers were cellulosic membranes but are produced differently; Dow fibers are regenerated cellulose from acetate, while Enka fibers are manufactured via the cuprammonium process.

The Enka fibers had a lower wall thickness, which, other things being equal, will allow for faster diffusion of glucose in and out of the sensor. Of course, a smaller wall thickness would increase the probability of mechanical damage but may not reduce the amount of bound Con A since most of the Con A binding is expected on the inside wall of the fiber. Details of preparing the fiber prior to use are given elsewhere. ${ }^{14}$

Con A was obtained from Pharmacia, while sodium fluorescein and the fluorescein isothiocyanate-dextran (FITC-dextran) were obtained from Sigma. ${ }^{3} \mathrm{H}$-Labeled Con A $(30 \mathrm{Ci} / \mathrm{mmol})$ was obtained from New England Nuclear. FITC-glycogen was a gift from Cardiac Pacemakers (St. Paul, MN). Biological-grade glutaraldehyde $(50 \% \mathrm{w} / \mathrm{w}$ solution) was obtained from Fisher Scientific.

\section{Periodate Oxidation}

Periodate oxidation was carried out according to the procedure of Sanderson and Wilson. ${ }^{13}$ Chopped fibers $(250 \mathrm{mg})$ with a final settled volume of $2 \mathrm{~mL}$ were used, and the concentration of the oxidant and the time of reaction were varied as indicated.

\section{Coupling of Spacer to the Activated Fiber}

A spacer group of 1,6-hexanediamine-glutaraldehyde was interposed between Con $A$ and the fiber. This was done in two steps. First, the diamine was attached to the dialdehyde formed by periodate oxidation, and glutaraldehyde was then coupled to the free amino end of the diamine. The extent of diamine coupling was monitored in the $\mathrm{pH}$ range 8.6-7.7, keeping the diamine concentration constant at $1 \%$ in phosphatebicarbonate buffer adjusted to the required $\mathrm{pH}$. The amount of free amino group on the fiber was determined by Ninhydrin assay. ${ }^{10}$ The ninhydrin reagent was prepared according to Moore. ${ }^{15}$ Alanine and $\varepsilon$-amino caproic acid were used as standards and an average value of $0.51 \pm 0.01 \mu \mathrm{mol} / \mathrm{OD}$ unit at $570 \mathrm{~nm}$ was used to calculate the micromoles of amino groups on the fiber.

Glutaraldehyde was attached to free amino groups with a $2 \%$ solution in phosphate buffer $(0.05 M, 1 M$ $\mathrm{NaCl}, \mathrm{pH}$ 7.2). The procedure has been described by Richards and Knowles. ${ }^{16}$ Reaction time was varied from 15 to $60 \mathrm{~min}$, and the remaining amino group was measured by the ninhydrin method as described above.

\section{Coupling of Con A to the Spacer}

The binding of Con $\mathrm{A}$ to the spacer was performed in the same buffer and at the same pH as the glutaraldehyde attachment step. Protein concentrations was determined before and in the supernatant after coupling either by absorbance at $280 \mathrm{~nm}$ (using an extinction coefficient of $\left.1.3 \mathrm{~cm}^{2} / \mathrm{mg}\right)^{14}$ or by the Lowry method ${ }^{17}$ using a protein standard solution obtained from Sigma.

In the initial set of experiments ${ }^{3} \mathrm{H}$-labeled Con $\mathrm{A}$ was used to determine the mass balance on the total amount of Con A used. Use of ${ }^{3} \mathrm{H}$-labeled Con A was a quick and easy technique to verify the protein assay obtained by the conventional assay methods. Following the optimization of the immobilization procedure, ${ }^{3} \mathrm{H}$ labeled Con A was not used.

The coupling reaction was carried out at $4^{\circ} \mathrm{C}$ with gentle mixing for a minimum of $24 \mathrm{~h}$ at a Con $\mathrm{A}$ concentration of $1 \mathrm{mg} / \mathrm{mL}$ and a total amount of 10 $\mathrm{mg}$ protein. Following immobilization, the fibers were washed with large amounts of coupling buffer and treated with $1 \%$ solution of sodium borohydride $\left(\mathrm{NaBH}_{4}\right)$ for $1 \mathrm{~h}$ at room temperature. A diagram outlining the coupling procedure is shown in Figure 1.

\section{Active Site Titration of Con A}

Fibers with bound Con A ( $250 \mathrm{mg}$ fibers dry weight) were washed with borate buffer $(0.02 M$ containing $0.5 \mathrm{M} \mathrm{NaCl}, \mathrm{pH} 6.3)$ to remove any bound saccharide and suspended in $3 \mathrm{~mL}$ phosphate buffer $(0.05 M, 0.15 M$ $\mathrm{NaCl}, \mathrm{pH}$ 7.2.). One milliliter of $40 \mu \mathrm{g} / \mathrm{mL}$ FITCdextran was added, and the suspension was gently shal.en for $\mathbf{3 0} \mathrm{min}$, time enough to attain equilibrium. The fibers were settled by centrifugation, and the amount of free FITC-dextran in the supernatant was measured using a Perkin Elmer fluorescence spectrometer with the excitation and emission monochromators set at 490 and $520 \mathrm{~nm}$, respectively. An identical experiment with untreated fibers yielded the total FITC-dextran concentration. The number of dextran binding sites was calculated using a simple, 


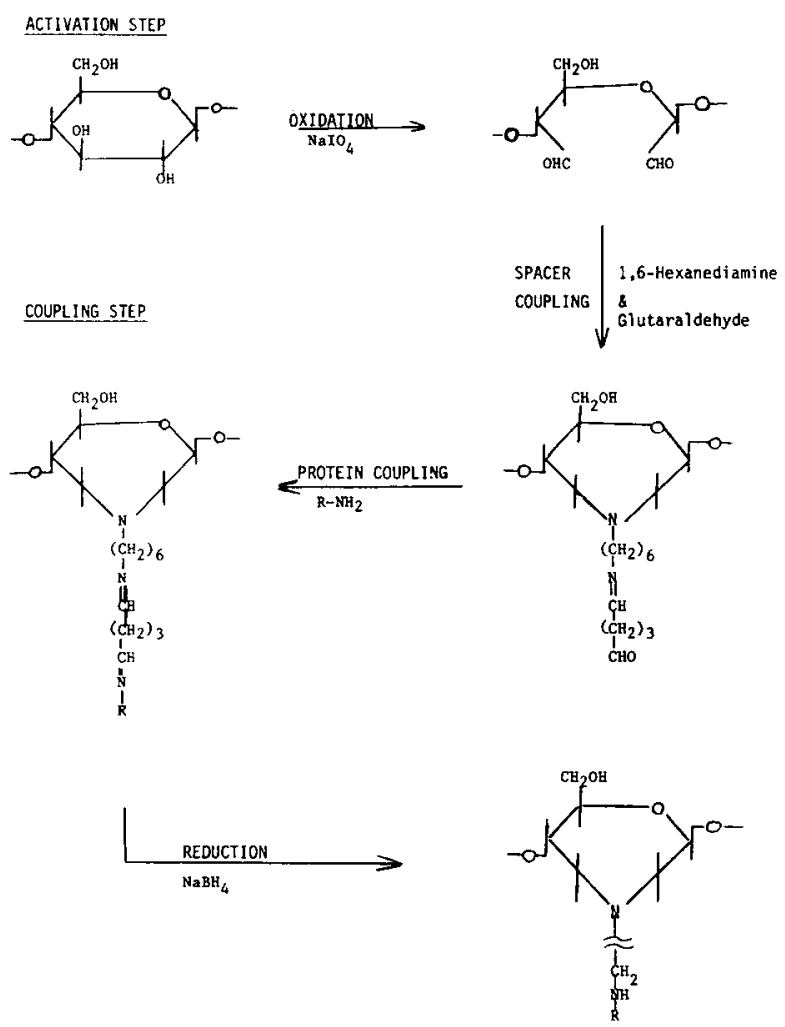

Figure 1. Chemistry of Con A Immobilization in Single Fibers.

monovalent binding equilibrium between bound Con A and dextran:

$$
C_{t}=\frac{1}{D_{f} K}\left(D_{t}-D_{f}\right)
$$

where $C_{t}$ is the total concentration of polysaccharide binding sites on the fiber (mol/L), $D_{t}$ and $D_{f}$ are, respectively, the total and free dextran concentrations $(\mathrm{mol} / \mathrm{L})$, and $K$ is the binding constant $(\mathrm{L} / \mathrm{mol})$. We used a value of $1.5 \times 10^{4}$ for $K .^{2}$ This value of the binding constant was obtained from a binding study between dextran in solution and Con A immobilized on Sepharose beads.

A molecular weight of 70,000 daltons for dextran and 26,000 for the Con A site were used.

\section{Con A Immobilization in Single Fibers}

Con A was immobilized on the inside wall of a single fiber under optimal conditions obtained from above experiments. Enka hollow fibers were prepared for immobilization according to the method described by Mansouri. ${ }^{14}$ The two ends of a single fiber ( $15 \mathrm{~cm}$ long) were sealed with epoxy cement onto the tips of two 23-gauge needles, and the reactant solutions were pushed through the lumen of the fiber (during which time the fiber was kept immersed in appropriate buffer with gentle agitation) using an infusion pump. The rate of infusion and the total volume of solution were varied to regulate the duration of each step. The integrity of the fiber was examined after every step of immobilization.

In the Con A coupling step, the following modifications were found to produce substantial improvements:

1. Con A was purified by affinity chromatography on a Sephadex G-75 column. ${ }^{14}$ Affinity chromatography of Con A, a simple, one-step procedure, was included to obtain Con A of well-defined affinity for reproducible results. Since many different sources of Con A are available, it is our belief that the above method would help to standardize the Con A used in coupling.

2. A slight back pressure during Con A infusion caused ultrafiltration of protein solution through the fiber membrane, which, when combined with $0.15 \%$ solution of glutaraldehyde in the external solution, resulted in the formation of a cross-linked layer of Con $A$ on the inside wall of the fiber.

Table I summarizes the steps involved in the immobilization of Con A onto the inside walls of a single hollow fiber.

Estimation of bound Con $A$ on single fibers was done by subtracting the total amount of Con A recovered after immobilization from the total amount applied. It was found that $10-15 \%$ of the initial amount of Con A was bound to a $15-\mathrm{cm}$ length of a single fiber.

The concentration of active Con $\mathrm{A}$ in a single fiber was quantitated on the basis of its performance when used as the transducer element of a glucose sensor. Briefly, a hollow cylindrical chamber was constructed from a suitable length of Con A-bound fiber. One side of the fiber was sealed with epoxy, and an optical fiber was centrally located from the other side and sealed in place. Fluorescence of FITC-dextran solution contained inside the above chamber was measured under conditions when all the dextran molecules are bound to Con $\mathrm{A}$ on the wall $\left(F_{\min }\right)$ and when all the dextran

Table I. Reaction steps for immobilizing Con A in a single hollow fiber.

\begin{tabular}{|c|c|c|c|c|c|}
\hline Reaction step & Reactant & Concentration & Medium & $\mathrm{pH}$ & Reaction time (h) \\
\hline Oxidation & Sodium periodate & $50 \mathrm{~m} M$ & Water & Neutral & 5 \\
\hline Spacer coupling & 1,6-Hexane diamine & $1 \%$ & Phosphate buffer & 8 & 5 \\
\hline Spacer coupling & Glutaraldehyde & $2 \%$ & Phosphate buffer $+\mathrm{NaCl}$ & 7.2 & 0.75 \\
\hline Immobilization & Con A & $1.0 \mathrm{mg} / \mathrm{mL}$ & & 7.2 & 24 \\
\hline Reduction & Sodium borohydride & $1 \%$ & 0.5M Phosphate & 8 & 1 \\
\hline Final wash & Borate buffer & $0.02 M$ & Borate buffer & 6 & $\frac{1}{2}$ \\
\hline
\end{tabular}


was free in solution inside the chamber $\left(F_{\max }\right)$. It has been shown that ${ }^{14}$

$$
\mu \mathrm{g} \text { Con } \mathrm{A} / \mathrm{cm} \text { fiber }=0.55\left(1.67 F_{\max } / F_{\min }-1\right)
$$

Assuming each Con A site to have a molecular weight of 26,000 daltons, the binding constant $K_{A}=15,000 M^{-1}$, and the inside diameter of the fiber is $0.2 \mathrm{~mm}$.

\section{Photomicrography}

Fluorescence microscopy was employed to detect Con A on hollow fibers using FITC-glycogen as a tracer. Con A-bound fibers were cut into pieces (1-2 $\mathrm{mm}$ in length) and suspended in $50 \mu \mathrm{g} / \mathrm{mL} \mathrm{FITC-}$ glycogen in phosphate-buffered saline. The fibers were observed at $40 \times$ by a fluorescence microscope (Vanox, Olympus).

\section{Mechanical Strength}

Fibers were examined to identify any major deterioration in the mechanical properties. The procedure was to hold a piece of fiber immersed in PBS with tweezers and bend it into a V-shape several times. Presence of cracks or deformations on the fiber at the point of stress (when examined under a microscope) was regarded as an unacceptable loss of fiber's mechanical integrity.

\section{Permeability Test}

The mass transfer properties of the fiber were evaluated by measuring the permeability of Con A-bound fiber and comparing it with that of an intact fiber. Sodium fluorescein was selected as the test solute, and the nonsteady-state method of Stevenson et al. ${ }^{18,19}$ was adopted. The optical system developed for the glucose sensor was used to monitor the diffusive transport of fluorescein into and out of the hollow fiber. Further details are given elsewhere. ${ }^{14}$

\section{RESULTS}

The major concern in this study was to maximize the amount of immobilized Con A on the fibers without deterioration of the physical properties of the fiber as a result of the structural modifications needed to develop active sites. Table II represents the effects of $\mathrm{NaIO}_{4}$ concentration and the duration of the reaction on the extent of Con A immobilization, the reactivity of immobilized Con A, and in a few cases the mechanical integrity of the resultant fibers. As expected, the highest yield was obtained with the largest concentration of periodate $(100 \mathrm{~mm})$ and the longest time $(20 \mathrm{~h})$. As a measure of comparison, the highest yield of bound Con A ( $32 \mathrm{mg} / \mathrm{g}$ fiber or $4 \mathrm{mg} / \mathrm{mL}$ settled fiber) obtained in this study is roughly $40 \%$ of the amount of Con A on commercially available Con A-Sepharose 4B gel
Table II. Effect of $\mathrm{NaIO}_{4}$ concentration and the time of oxidation on the amount of Con $\mathrm{A}$ bound and its reactivity.

$250 \mathrm{mg}$ chopped fibers were reacted with the indicated concentrations of $\mathrm{NaIO}_{4}$ for various durations. 1,6-Hexanediamine was coupled to the oxidized fibers at $\mathrm{pH} 8.6 \mathrm{using} 1 \%$ solution and $5 \mathrm{~h}$ reaction time. Following glutaraldehyde attachment at $\mathrm{pH} 7.2$ for $1 \mathrm{~h}$ using $2 \%$ solution, Con A was coupled at $\mathrm{pH} 7.2$ and $4^{\circ} \mathrm{C}$ for $24 \mathrm{~h}$ at a Con $A$ concentration of $1 \mathrm{mg} / \mathrm{mL}$ and a total of $10 \mathrm{mg}$ protein. All reactions were carried out with continuous agitation and at room temperature except for Con $A$ coupling as indicated above. Amount of bound Con $\mathrm{A}$ was taken as the difference between the initial and the final amounts of protein in solution. Active Con $A$ was estimated as described in the text. The values reported are the average of four measurements with a spread of $15 \%$.

\begin{tabular}{cccc}
$\begin{array}{c}\text { Concentration } \\
\text { of } \mathrm{NaIO}_{4} \\
(\mathrm{~m} M)\end{array}$ & $\begin{array}{c}\text { Reaction } \\
\text { time } \\
(\mathrm{h})\end{array}$ & $\begin{array}{c}\text { Con A } \\
\text { bound } \\
(\mathrm{mg} / \mathrm{g} \text { fiber })\end{array}$ & $\begin{array}{c}\text { Active } \\
\text { Con A } \\
(\mathrm{mg} / \mathrm{g} \text { fiber })\end{array}$ \\
\hline 10 & 5 & 0 & $\mathrm{ND}^{\mathrm{a}}$ \\
& 8 & 2.8 & $\mathrm{ND}$ \\
25 & 20 & 2.6 & $\mathrm{ND}$ \\
& 5 & 0 & $\mathrm{ND}$ \\
& 8 & 4.0 & $<1.0$ \\
50 & 20 & 7.2 & 1.5 \\
& 5 & 15.2 & 8.0 \\
100 & 8 & 18.0 & 8.4 \\
& 20 & 18.0 & - \\
& 5 & 15.2 & $\mathrm{ND}^{\mathrm{b}}$ \\
& 8 & 22.4 & $\mathrm{ND}$ \\
& 20 & 32.0 & 13.2 \\
\hline
\end{tabular}

a Preliminary experiments indicated that at low levels of Con A coupling the fibers will not have enough sensitivity as a glucose sensor.

${ }^{b}$ At these high concentrations of $\mathrm{NaIO}_{4}$, considerable structural damage to the fiber could be seen under the microscope.

$(10 \mathrm{mg} / \mathrm{mL}$ gel). The differences in the yield may be related to the fact that ours is a multistep coupling procedure, whereas the commercial process is onestep activation followed by direct coupling Con A to the matrix.

As indicated in Table $\mathrm{II}, 100 \mathrm{mM} \mathrm{NaIO}$ oxidation was detrimental to the fiber's mechanical strength, whereas $10 \mathrm{~m} M$ and $25 \mathrm{~m} M$, though less rigorous than the former, were inadequate in terms of the sensitivity required of the affinity sensor. However, $50 \mathrm{~m} M$ and $5 \mathrm{~h}$ reaction time was consistently found to yield optimal amounts of bound Con A with minimal or no structural damage.

\section{Spacer Coupling: pH Effect}

The use of spacer groups to make the critical chemical groups involved in the affinity interaction distal to the matrix is well known, ${ }^{20}$ and in the case of high-molecular-weight proteins with systems of low binding affinity, minimal steric hindrance posed by the use of spacer group seems quite important. From molecular dimensions, we estimate 1,6-hexanediamine-glutaraldehyde spacer to be about $15 \AA$ long and is formally 
equivalent to the diisocyanate-glutaraldehyde used by Chen and $\mathrm{TsaO}^{21}$ to couple enzymes to cellulose beads. Results shown in Table III reveal that at higher $\mathrm{pH}$ substantial amounts of amino groups are attached to the fibers. Once again, single fiber experiments and the subsequent examination of the fiber indicated some deterioration of the fiber above $\mathrm{pH}$ 8.0. This was surprising since the cellulose fiber is expected to be stable at such a low alkaline $\mathrm{pH}$. Still, the combination of periodate oxidation and higher $\mathrm{pH}$ may be detrimental to the fiber in a manner not readily understood. We observed, as did Sanderson and Wilson, ${ }^{13}$ that the fibers acquired a slight yellow color following 1,6hexanediamine coupling.

We therefore chose $\mathrm{pH} 8$ as the optimal $\mathrm{pH}$ for the 1,6-hexanediamine coupling step.

Table III also indicates that the extent of Con A coupling to the fibers parallels the amino group concentration on the fibers, and this leads us to believe that the glutaraldehyde coupling is monofunctional. Howerer, examination of Table IV shows that glutaraldehyde coupling may be bifunctional. For example, doubling the amount of amino groups reacting with glutaraldehyde produces only minor increases in the amount of Con A coupled to the fiber, which indicates cross-linking of vicinal amino groups by glutaraldehyde.

Table III. Effect of $\mathrm{pH}$ on the extent of 1,6-hexanediamine coupling.

The experimental conditions were identical to the ones described in Table II except for periodate oxidation, which was carried out for $5 \mathrm{~h}$ at $50 \mathrm{~m} M$ concentration. Amino groups were assayed using ninhydrin reagent. Unreacted fibers and oxidized fibers had negligible amounts of amino groups. The range of amino group values indicates the highest and the lowest in a set of four measurements. Other values are average of four determinations with a spread of $13 \%$.

\begin{tabular}{lccc}
$\mathrm{pH}$ & $\begin{array}{c}\text { Amino groups } \\
\text { bound } \\
\text { (mol/g fiber) }\end{array}$ & $\begin{array}{c}\text { Con A } \\
\text { bound } \\
\text { (mg/g fiber) }\end{array}$ & $\begin{array}{c}\text { Active } \\
\text { Con A } \\
\text { (mg/g fiber) }\end{array}$ \\
\hline 8.6 & $60-70$ & 18.0 & 7.2 \\
8.2 & $52-56$ & 15.6 & 11.6 \\
7.7 & $39-43$ & 11.6 & 6.0 \\
\hline
\end{tabular}

Table IV. Effect of glutaraldehyde reaction time on the extent of Con $\mathrm{A}$ binding to the fibers.

Periodate oxidation and 1,6-hexanediamine coupling were carried out under the respective optimal conditions. All other reaction conditions were the same as in Table II. The values reported are the average of a set of four determinations with a spread of about $12 \%$.

\begin{tabular}{cccc}
$\begin{array}{c}\text { Reaction } \\
\text { time } \\
(\mathrm{min})\end{array}$ & $\begin{array}{c}\text { Amino groups } \\
\text { reacted } \\
(\%)\end{array}$ & $\begin{array}{c}\text { Con A } \\
\text { bound } \\
\text { (mg/g fiber) }\end{array}$ & $\begin{array}{c}\text { Active } \\
\text { Con A } \\
\text { (mg/g fiber) }\end{array}$ \\
\hline 15 & 16.2 & 15.2 & 7.2 \\
30 & 33 & 18.0 & 7.2 \\
45 & 50 & 18.4 & 9.2 \\
60 & 66.7 & 17.2 & 8.4 \\
\hline
\end{tabular}

Dincer ${ }^{22}$ has shown that glutaraldehyde is an effective reagent to block excess amino groups on the surface of polymethylmethacrylate beads. Even so, it appears that the unreacted amino groups on the fibers might confer anion exchange properties to the cellulose matrix around neutral $\mathrm{pH}$. Since the Con A coupling step as well as the washing of the fibers following Con A coupling were carried out with buffers of high ionic strength $(0.05 M$ phosphate, $1 M \mathrm{NaCl}, \mathrm{pH} 7.2)$ this interference is expected to be minimal. However, in the future fabrication of the affinity sensor we plan to use adipoyl dihydrazide in place of 1,6-hexanediamine, and this is expected to result in an uncharged cellulose matrix at neutral $\mathrm{pH}$. Second, with single fibers, amino groups span the entire wall thickness of the fiber while Con A couples only to the layers contiguous to the lumen. This would reduce the amount of nonspecifically adsorbed Con A. Finally, since the estimates of the bound Con $\mathrm{A}$ are far less than the theoretical value based on the moles of glutaraldehyde-coupled amine groups located on the fibers, cross-linking by glutaraldehyde is indicated, which is beneficial in terms of charge neutralization as discussed above.

In the case of single fibers, we used $45-60 \mathrm{~min}$ as the optimal for glutaraldehyde coupling reactions.

More Con A was immobilized on the inside wall of the single hollow fiber by ultrafiltration of the protein and subsequent cross-linking with glutaraldehyde. The thickness of the Con A deposit was related to the concentration of glutaraldehyde in solution outside the fiber and the flowrate of Con A solution in the hollow fiber. At the input flowrate of $0.15 \mathrm{~mL} / \mathrm{h}$, a thin deposit visible under the microscope was formed at concentrations as low as $0.01 \%(\mathrm{w} / \mathrm{v})$ glutaraldehyde, while at $0.2 \%$ concentration the fiber was found clogged with the deposit. The thickness of the deposit was not uniform throughout the fiber's length; it was thinner near the input and became thicker close to the end of the fiber. This unevenness was attributed to a slowdown in the axial flowrate as a result of the ultrafiltration of the Con A solution through the wall of the hollow fiber. Actually, less than half of the injected Con A solution was recovered, indicating that the residence time near the end of the fiber was approximately twice as high as that close to the entrance.

Excessive Con A cross-linking, however, had an adverse effect on the sensor performance in terms of the maximum signal change and response time, as shown in Table $\mathrm{V}$. The signal change (ratio of the bound to total fluorescence) is related to the amount of active Con $A$ in the fiber. The slight decrease in the signal change at $0.05 \%(w / v)$ glutaraldehyde was presumably due to the inaccessibility of Con A molecules to dextran as a result of an excessive protein crosslinking. The level of Con A cross-linking at $0.01 \%$ glutaraldehyde was found the most suitable based on the performances on the tested sensors. 
Table V. Effects of glutaraldehyde concentration on the extent of Con A cross-linking in hollow fibers.

\begin{tabular}{lccc}
\hline Glutaraldehyde & $\begin{array}{c}\text { Maximum } \\
\text { Change in } \\
\text { permeability } \\
(\%)\end{array}$ & $\begin{array}{c}\text { signal change } \\
\text { in resulting } \\
\text { sensor }^{\mathrm{b}} \\
(\%)\end{array}$ & $\begin{array}{c}\text { Amount of } \\
\text { immobilized } \\
\text { Con A } \\
(\mu \mathrm{g} / \mathrm{cm} \text { fiber })\end{array}$ \\
\hline 0 & 40 & 25 & 0.67 \\
0.005 & - & 49 & 1.3 \\
0.01 & 90 & 86 & 6.0 \\
0.05 & 220 & 84 & 5.2 \\
0.2 & Fiber & - & - \\
\hline
\end{tabular}

\footnotetext{
a Determined from $\left(P_{0}-P\right) / P_{0} \times 100$, where $P$ is the measured permeability and $P_{0}$ is the permeability of an intact fiber.

${ }^{\mathrm{b}}$ Maximum signal change is equal to $100\left(1-F_{\min } / F_{\max }\right)$, where $F_{\min }$ is the sensor output (in the form of fluorescence) at zero sugar and $F_{\max }$ is the corresponding sensor output at the highest sugar concentration used.
}

\section{Active Site Titration of Bound Con A}

The efficiency of the immobilization was tested by estimating the concentration of dextran binding sites on the fibers assuming monovalent binding between dextran and the Con A site. In all cases except one, $40-50 \%$ of the Con $\mathrm{A}$ on the fiber was active, the balance assumed to be inactive toward dextran binding. The loss of dextran binding ability may have been due to (1) inactivation of Con A molecule during the coupling process or (2) the involvement (directly or otherwise) of the carbohydrate binding site of Con A in the coupling reaction.

A more likely explanation is that our assumption of monovalent binding between Con $\mathrm{A}$ and dextran is probably not strictly correct. For example, Con A is tetrameric at neutral $\mathrm{pH}$ and dextran of 70,000 daltons has been estimated to have about 15 nonreducing chain ends, ${ }^{14}$ each capable of binding to Con A. Thus, we believe our estimate of the concentration of active sites on the fibers to be a lower bound.

The extent of Con A denaturation during coupling is difficult to evaluate. However, use of excess glucose during the Con A coupling step (use of excess substrate is expected to protect the substrate binding site from any possible involvement in the coupling reaction) showed no significant improvement in active site concentration.

To place our results in perspective, we used methyl mannoside to estimate the active site population in a commercial preparation of Con A bound to Sepharose $4 \mathrm{~B}$ and found approximately $70 \%$ of bound Con $\mathrm{A}$ to be active.

In the case of single hollow fibers, bound Con A was estimated, as mentioned before, with an average value of $6.5 \mu \mathrm{g} / \mathrm{cm}$ fiber. Furthermore, active Con A was found to range between 2 and $3 \mu \mathrm{g} / \mathrm{cm}$ fiber.

The mechanical strength of single fibers was undiminished under optimal condition of $\mathrm{NaIO}_{4}$ oxidation (50m $M$ and $5 \mathrm{~h}$ ), 1,6-hexanediamine coupling (1\% solution of $\mathrm{pH} 8.0$ and $5 \mathrm{~h}$ ), and glutaraldehyde attachment ( $2 \%$ solution, $\mathrm{pH} 7.2$ and $45 \mathrm{~min}$ ). On the other hand, permeability of single fibers to small molecules (measured using sodium fluorescein) was mainly affected by the extent of Con A cross-linking when glutaraldehyde was used in the external solution during Con A coupling. For example, $0.2 \%(\mathrm{w} / \mathrm{v})$ glutaraldehyde concentration in the external solution resulted in a thick deposit of Con A on the fiber and clogged it. A $0.01 \%$ solution was most effective in producing crosslinked Con A (which provided long-term stability to bound Con A) without any loss of permeability.

\section{DISCUSSION}

The coupling steps outlined in this study are quite similar to other reports. ${ }^{9}$ However, the efficiency of immobilization is better in our case, and we estimate $21 \mu \mathrm{g}$ active Con $\mathrm{A} / \mathrm{cm}^{2}$ inside surface area of the fiber compared to a value of $0.3 \mu \mathrm{g} / \mathrm{cm}^{2}$ active arginase obtained in an earlier work. ${ }^{9}$ Our estimate is based on an average of $2 \mu \mathrm{g}$ active Con $\mathrm{A} / \mathrm{cm}$ fiber and an i.d. of $0.03 \mathrm{~cm}$. The low yield reported ${ }^{9}$ earlier may be due to the fact that they used $\mathrm{pH} 5.0$ during the attachment of 3,3'-diaminodipropylamine as a spacer to the periodate-oxidized fibers. At that comparatively low $\mathrm{pH}$, the alkylamines are positively charged, and the extent of reaction with the aldehyde groups on the fiber may be small. It should be pointed out that though the use of $\mathrm{pH} 5.0$ for the alkylamine coupling step is an acceptable procedure, it is expected to give poor yield of active groups. Since our aim was to maximize the amount of bound Con A, it is our belief that our procedure is a required improvement.

Visual and microscopic examinations confirm the essential findings ${ }^{9}$ that the structural integrity of the fibers is maintained during the coupling procedure. Glucose affinity sensors prepared from our Con Aimmobilized single fibers have been found to yield satisfactory and reproducible results in both in vitro and in vivo estimation of glucose concentration in plasma and blood. ${ }^{23}$

\section{CONCLUSIONS}

A procedure for immobilizing Con $A$ on the inside wall of a single cellulosic fiber is described. The following solutions were passed through in succession to obtain the results reported in this study: (1) sodium periodate $(50 \mathrm{~m} M)$ for $5 \mathrm{~h},(2)$ 1,6-hexanediamine (1\%) at $\mathrm{pH} 8$ for $5 \mathrm{~h},(3)$ glutaraldehyde $(2 \%)$ at $\mathrm{pH} 7.2$ for 45-60 min, (4) Con A (1 mg/mL) at pH 7.2 and $4^{\circ} \mathrm{C}$ for $24 \mathrm{~h}$ minimum with $0.01 \%$ glutaraldehyde in the outside solution, and (5) sodium borohydride (1\%) at $\mathrm{pH} 8$ for $1 \mathrm{~h}$.

In summary, the above procedure yields typically $5-6 \mu \mathrm{g}$ Con $\mathrm{A} / \mathrm{cm}$ fiber, of which $50-60 \%$ is active. 


\section{References}

1. J. S. Schultz and G. Sims, Biotechnol. Bioeng. Symp., 9, 65 (1979).

2. J. S. Schultz, S. Mansouri, and I. Goldstein, Diabetes Care, 5(3), 245 (1982).

3. W. H. Scouten, Affinity Chromatography, Chemical Analysis, Vol. 59 (Wiley, New York, 1981).

4. J. Turkova, Affinity Chromatography (Elsevier, Amsterdam, 1978).

5. J. A. Jackson, H. R. Halvorson, J. W. Furlong, K. D. Lucast, and J. D. Shore, Pharmacol. Exp. Ther, 209, 271 (1979).

6. F. Dumler, P. Singh, C. E. Jackson, K. R. Kini, A. M. Samhouri, H. R. Halvorson, and J. D. Shore, Am. Soc. Artif. Intern. Organs, 4, 70 (1981).

7. G. Mazzola and G. Vecchio, Int. J. Artif. Organs, 3, 120 (1980).

8. P. G. Pietta, D. Agnellini, G. Mazzola, G. Vecchio, S. Colombi, and G. Bianchi, Enzyme Engineering, Vol. 5 (Plenum Press, New York, 1980), p. 235.

9. V. Rossi, A. Malinverni, and L. Callegaro, Int. J. Artif. Organs, 4, 102 (1981).
10. T. H. Finlay, V. Troll, M. Levy, A. J. Johnson, and L. Hodgins, Anal. Biochem., 87, 77 (1978).

11. B. Svensson, FEBS Lett., 29, 167 (1973).

12. M. Wilchek and T. Miron, Meth. Enz., 34, 72 (1974).

13. C. J. Sanderson and D. V. Wilson, Immunology, 20, 101 (1971).

14. S. Mansouri, Ph.D. Thesis, The University of Michigan, Ann Arbor, MI, 1983.

15. S. Moore, J. Biol. Chem., 243, 6281 (1968).

16. F. M. Richards and J. R. Knowles, J. Mol. Biol., 37, 231 (1968).

17. T. G. Cooper, The Tools of Biochemistry, (Wiley, New York, 1977), p. 53.

18. J. F. Stevenson, AIChE, 20, 461 (1974).

19. J. F. Stevenson, M. A. Von Deak, M. Weinberg, and R. W. Schuette, AIChE, 21, 1192 (1975).

20. P. Cuatrecasas, J. Biol. Chem., 245, 3059 (1970).

21. L. F. Chen and G. T. Tsao, Biotechnol. Bioeng,, 19, 1463 (1977).

22. A. K. Dincer, "Covalent Coupling of Heparin to Synthetic Polymer Surfaces," Ph.D. Dissertation, MIT, 1977.

23. S. Mansouri and J. S. Schultz, Bio/Technol, October 1984. 